\title{
Age moderates the association between psychological distress and engagement in mindfulness among cancer patients and survivors: A population-based study
}

\section{Anao Zhang, Rita Hu, Kaipeng Wang \& Erin Peregine Antalis}

To cite this article: Anao Zhang , Rita Hu , Kaipeng Wang \& Erin Peregine Antalis (2020) Age moderates the association between psychological distress and engagement in mindfulness among cancer patients and survivors: A population-based study, Journal of Psychosocial Oncology, 38:5, 513-526, DOI: $10.1080 / 07347332.2020 .1764158$

To link to this article: https://doi.org/10.1080/07347332.2020.1764158

曲 Published online: 25 May 2020.

Submit your article to this journal $₫$

山 Article views: 190

Q View related articles $\sqsubset$

View Crossmark data $[\pi$

Citing articles: 4 View citing articles $匚$ 


\title{
Age moderates the association between psychological distress and engagement in mindfulness among cancer patients and survivors: A population-based study
}

\author{
Anao Zhang, $\mathrm{PhD}^{\mathrm{a}}$ (D), Rita $\mathrm{Hu}, \mathrm{MSW}^{\mathrm{a}}$, Kaipeng Wang, $\mathrm{PhD}^{\mathrm{b} *}$ (D), and \\ Erin Peregine Antalis, $\mathrm{PhD}^{\mathrm{C}}$ \\ ${ }^{a}$ School of Social Work, University of Michigan, Ann Arbor, MI, USA; ${ }^{b}$ School of Social Work, \\ Texas State University, San Marcos, TX, USA; 'Department of Internal Medicine, University of \\ Michigan Medical School, Ann Arbor, MI, USA
}

\begin{abstract}
Purpose: We aim to evaluate the relationship between psychological distress and engagement in mindfulness among a national representative sample of cancer survivors.

Sample and design: Using the 2017 National Health Interview Survey, our final analytical sample included 3068 participants who reported having been diagnosed with cancer.

Methods and analysis: We used logistic regression analysis to assess the association and to test age as a moderator. We also conducted follow-up analysis using Fisher's $r$-to- $z$ transformation. All analyses were adjusted for complex sample weights.

Findings: Cancer survivors who had subclinical and clinical psychological distress were more likely to engage in mindfulness, $\mathrm{OR}=1.59,95 \% \mathrm{Cl}[1.24,2.02]$ and $\mathrm{OR}=1.45,95 \% \mathrm{Cl}$ $[1.02,2.05]$, respectively. Age significantly moderated such association with the relationship much stronger among those who are younger (younger than 65 years old) than those who are older (65 years or older), $b=0.97,95 \% \mathrm{Cl}[0.95,0.99]$.

Conclusions: The relationship between psychological distress and engagement in mindfulness differs significantly by a survivor's age. Psychosocial oncological providers need to account for a survivor's age when delivering mindfulness based care to address psychological distress.

Implications: Providers should be mindful of a survivor's age when recommending mindfulness oriented practices.
\end{abstract}

\section{KEYWORDS}

Age; mindfulness; moderation; psychological distress; survivorship

\section{Introduction}

The national cancer survival rate has increased by $20 \%$ over the past three decades, with an estimated 15.5 million cancer survivors in the United States in 2016. ${ }^{1}$ The number is expected to increase to 20.3 million by

CONTACT Anao Zhang, PhD zhangan@umich.edu $\mathrm{E}$ School of Social Work, University of Michigan, Office 3704, 1080 S. University Ave., Ann Arbor, MI 48109, USA.

*Present address: Graduate School of Social Work, University of Denver, Denver, CO, USA.

(C) 2020 Taylor \& Francis Group, LLC 
2026. As the number of cancer survivors significantly increases over the years, cancer survivors' psychosocial wellbeing starts to receive enhanced attention. Psycho-oncology literature has consistently documented that cancer survivors, including patients receiving active treatment and those who are receiving survivorship-oriented care, are at high risk of experiencing psychological distress such as depression, anxiety, fear of recurrence and mortality, and poor quality of life. ${ }^{2-4}$ More importantly, the prevalence of psychosocial challenges is consistent across the life span of cancer population. ${ }^{2,5,6}$

Among various psychosocial challenges that confront cancer survivors, psychological distress is one of the most prevalent and debilitating challenges for cancer survivors. High level of psychological distress is associated with sleep disturbance, functional disability, poor care management, and low quality of life, all of which are detrimental to the treatment and recovery of cancer survivors. ${ }^{7,8}$ To alleviate psychological distress, the field has identified that engaging in mindfulness practice has positive effects on reducing cancer survivors' psychological distress across all age groups. ${ }^{3,9,10}$ Mindfulness practice is defined as a receptive attention to and awareness of present events and experience. ${ }^{8}$ Studies have suggested that mindfulness practice can be effective for psychological distress because it activates an individual's secondary control strategies. Secondary control, according to the Motivational Theory of Life Span Development (henceforth as the Motivational Theory), is the strategy that focuses on one's internal and motivational processes to minimize the emotional reactivity and obstacles created by losses of one's primary control, that is the direct control over one's body and environment. ${ }^{11}$ The Motivational Theory frames human development as an action cycle of striving for primary control capacity while engaging in secondary control to compensate for any losses/decreases in the primary control.

Most cancer survivors regardless of age have experienced losses of primary control including impaired health and functional status both during and after their treatment. Despite its benefits for cancer survivors, only focusing on regaining primary control of their body through cancer treatment would result in greater psychological distress. ${ }^{12}$ That is because losing primary control of one's body during and after cancer treatment is almost inevitable. Therefore, focusing on the highly unlikely regaining of primary control would further instigate feelings of frustration, depression, and anxiety among cancer survivors. Engagement in mindfulness, on the other hand, activates survivors' secondary control strategies so that they can better cope with the current situation to reduce psychological distress. ${ }^{10}$ For example, instead of struggling with maintaining primary control, a cancer survivor may utilize mindfulness to activate causal attribution strategies (a type of secondary control strategy) to avoid getting frustrated over the 
inability to complete as many daily tasks as one can before cancer diagnosis. In general, mindfulness practice is an active process of restructuring one's goals, rather than a passive reflection of failure and loss.

To date, ample empirical studies have documented the significant positive effect meditation has on psychological distress among cancer survivors. ${ }^{13-15}$ Specifically, studies have reported that mindfulness-based practices significantly reduce cancer survivors' psychological distress and stress level immediately post-treatment and at six-month follow-up. ${ }^{16,17}$ Furthermore, the effects of mindfulness-based practices on cancer survivors extend beyond distress reduction and further improve cancer survivors' general wellbeing and overall quality of life. ${ }^{18,19}$ Most compellingly, several systematic reviews and meta-analyses have consistently documented the positive effect of mindfulness-based practices on cancer survivors' psychological distress and overall quality of life across disease stages and diagnoses. $^{3,20,21}$

Despite the evidence that mindfulness practice is overall effective for cancer survivors, engagement in mindfulness is likely to vary among different age groups because the "primary - secondary control balance" changes across the life span. According to the Motivational Theory, even though the strive for primary control remains consistently high across lifespan, an individual's primary control capacity peaks during adolescence and young adulthood, plateaus during midlife, and decreases in later life. ${ }^{22}$ In order to maintain high primary control, individuals activate secondary control strategies to compensate for primary control as they age.

When cancer survivors experience a loss of primary control due to their medical conditions, older survivors have already better adapted to the decrease of primary control (due to aging) than their younger counterparts through activating various secondary control strategies. ${ }^{11,23,24}$ However, younger and middle aged adult survivors were at the peak of their primary control capacity before a cancer diagnosis, thus their secondary control capacities have not fully developed. Consequently, younger and middle aged adult cancer survivors may find mindfulness a more helpful method to activate secondary control than their older counterparts do.

Despite the literature on the association between psychological distress and engagement in mindfulness, few studies have investigated whether such relationship is consistent across age groups. Since most cancer survivors are 65 years or older, it is important to examine to what extent mindfulness practice (a secondary control activator) is associated with psychological distress among cancer survivors across ages. Therefore, using a national representative sample, we aim to: (1) evaluate the relationship between psychological distress and engagement in mindfulness among cancer survivors; and (2) assess if age moderates the relationship between 
psychological distress and engagement in mindfulness among cancer survivors. Specifically, we test the following hypotheses: (1) there is a significant association between psychological distress and engagement in mindfulness among cancer survivors; and (2) age moderates the relationship between psychological distress and engagement in mindfulness among cancer survivors.

\section{Methods}

\section{Data source}

This study used data from the 2017 National Health Interview Survey (NHIS.) ${ }^{25}$ NHIS is an annual cross-sectional survey study that collects demographics and health-related information through in-person household interviews from a nationally representative sample of US non-institutionalized civilians (exclude long-term care, hospital and correctional facilities). The 2017 NHIS contains 32,617 households (response rate 66.5\%). This study's sample included 26,742 adults (response rate 80.7\%) who responded to NHIS's adult core survey that asked adults about their history of cancer diagnoses. The final analytic sample included a sample of $n=3068$ participants who reported having been diagnosed with cancer. For our regression analysis, because we used weights to account for complex sampling of NHIS, we used listwise deletion, resulting a final regression analytical sample of $n=2373$ ( $77.34 \%$ of the eligible sample).

\section{Focal variables}

Psychological distress was measured by the Kessler's K6 scale. ${ }^{26}$ Participants responded to six questions using a 5-point Likert scale $(1=$ all of the time to $5=$ None of the time). Items of the K6 scale are: during the past 30 days, about how often did you feel (a) nervous? (b) hopeless? (c) restless or fidgety? (d) so depressed that nothing could cheer you up? (e) that everything was an effort? ( $f$ ) worthless. The scale was recoded $(0=$ none of the time to $4=$ all of the time) and summed up (ranging from 0 to 24 ) with higher score indicating worse psychological distress. The variable was recoded into " $0=$ no distress at all," " $1=$ psychological distress, subclinical" (1-12) that is having some distress symptoms but does not meet clinical diagnostic criteria, and " $2=$ psychological distress, clinical" (13-24), that is meets clinical diagnostic criteria and demands specialized care. ${ }^{27}$ The study reported satisfactory internal consistency with Cronbach's alpha $=0.94$. 


\section{Engagement in mindfulness}

Engagement in mindfulness is measured by a survivor reported frequency of practicing mindfulness related activities over the past 12 months. From a list of 12 activities, participants were asked "over the past 12 months, did you use or practice the following activities?" Participants select $1=$ yes, or $0=$ no to indicate if they used or practiced these activities over the past 12 months. The 12 activities are: mantra meditation; mindfulness meditation; spiritual meditation; guided imagery; progressive relaxation; yoga with breathing exercise; yoga with meditation; Tai Chi with breathing exercise; Tai Chi with meditation; Chi Gong with breathing exercise; Chi Gong with meditation; and any other mindfulness or meditation involved activities. A composite score was summed to indicate engagement in mindfulness, ranging from 0 to 12 with higher score indicating greater engagement. Given the distribution of the variable, it was dichotomized into " $0=$ no engagement in mindfulness practice" and " $1=$ some engagement in mindfulness practice."

\section{Covariates}

This study controlled for demographic variables including: age in years, gender, race, age of first cancer diagnosis, marital status, education background, and family income. We also controlled for whether a participant had access to home care $(1=$ yes, $0=$ no $)$ and if a participant worried about medical bills $(1=$ yes, $0=$ no), as both variables are important correlates to psychological distress.

\section{Statistical methods}

All data analyses were performed in $\mathrm{R}$ software version 3.5 using the "survey" package. The "survey" package is one of the statistical packages recommended by the NHIS dataset that produces robust variance estimates for analyzing complex samples while incorporate appropriate sampling weights for subgroup analysis. We used logistic regression analysis to assess the association between psychological distress and engagement in mindfulness while controlling for important covariates. To account for the effect of multistage complex sampling design, all regression analyses used person level weight and robust variance estimation for subgroup data analysis. Moderation analysis was conducted by creating an interaction term of mean centered "(dichotomized) engagement in mindfulness" and "age" and testing the significance of the interaction term. Follow-up analysis using Fisher's $r$-to- $z$ transformation, with adjustment for survey weights, was used to evaluate the difference in associations between engagement in 
mindfulness (raw score as a continuous variable) and psychological distress (raw score as a continuous variable) at subgroups of the moderator (age, being dichotomized into those who are younger than 65 years old versus those who are 65 years or older. $)^{28}$ We chose 65 years old as the age cutoff point as this is the traditional threshold between older and younger Americans. ${ }^{29}$ For all statistical analyses, we used a conventional alpha level of $p<0.05$.

\section{Results}

All descriptive statistics were weight adjusted unless otherwise specified. Cancer survivors' age $(N=3068)$ averaged at 66.34 years old $(S D=14.96)$ and their initial cancer diagnosis age was $52.95(S D=18.83)$ on average. About half of them were female (58.23\%), married (47.5\%) and most being Hispanic (89.74\%). Participants' engagement in mindfulness averaged in 0.52 times last year $(76 \%$ of the participants did not engage in mindfulness at all). Participants' psychological distress scores averaged at 5.31 $(S D=0.13)$, ranging from 0 to 24 . Other demographic information are presented in Table 1.

Holding other covariates constant, psychological distress was positively and significantly associated with engagement in mindfulness (Model 1 in Table 2). Comparing with cancer survivors who had no psychological distress, survivors who had psychological distress, subclinical were 1.6 times more likely to engage in mindfulness, $O R=1.59, p<0.001$. Cancer survivors who had psychological distress, clinical, were 1.45 times more likely to engage in mindfulness, $O R=1.45, p<0.05$. In addition to Model 1 and 2 (Table 2) tested the moderating effect of age on the association between psychological distress and engagement in mindfulness. After entering the interaction term (engagement in mindfulness $\times$ age), age significantly moderated the association between psychological distress and engagement in mindfulness among those who were clinically distressed versus those who were not distressed at all, $O R=0.97, p<0.05$.

The association between being clinically distressed and participation in mindfulness differs, i.e., is moderated, by age. More specifically, as cancer survivors become younger, the association between psychological distress and participation in mindfulness becomes weaker. Subgroup analysis (presented in Model 3 and 4 in Table 2) indicated that, for younger cancer survivors, those who were subclinically and clinically distressed were more likely to engage in mindfulness than those with no distress, $O R=1.49$, $p<0.05$ and $O R=1.70, p<0.05$, respectively. In comparison, for older cancer survivors, only those who were subclinically distressed reported significantly higher odds of engagement in mindfulness, $O R=1.65, p<0.01$, 


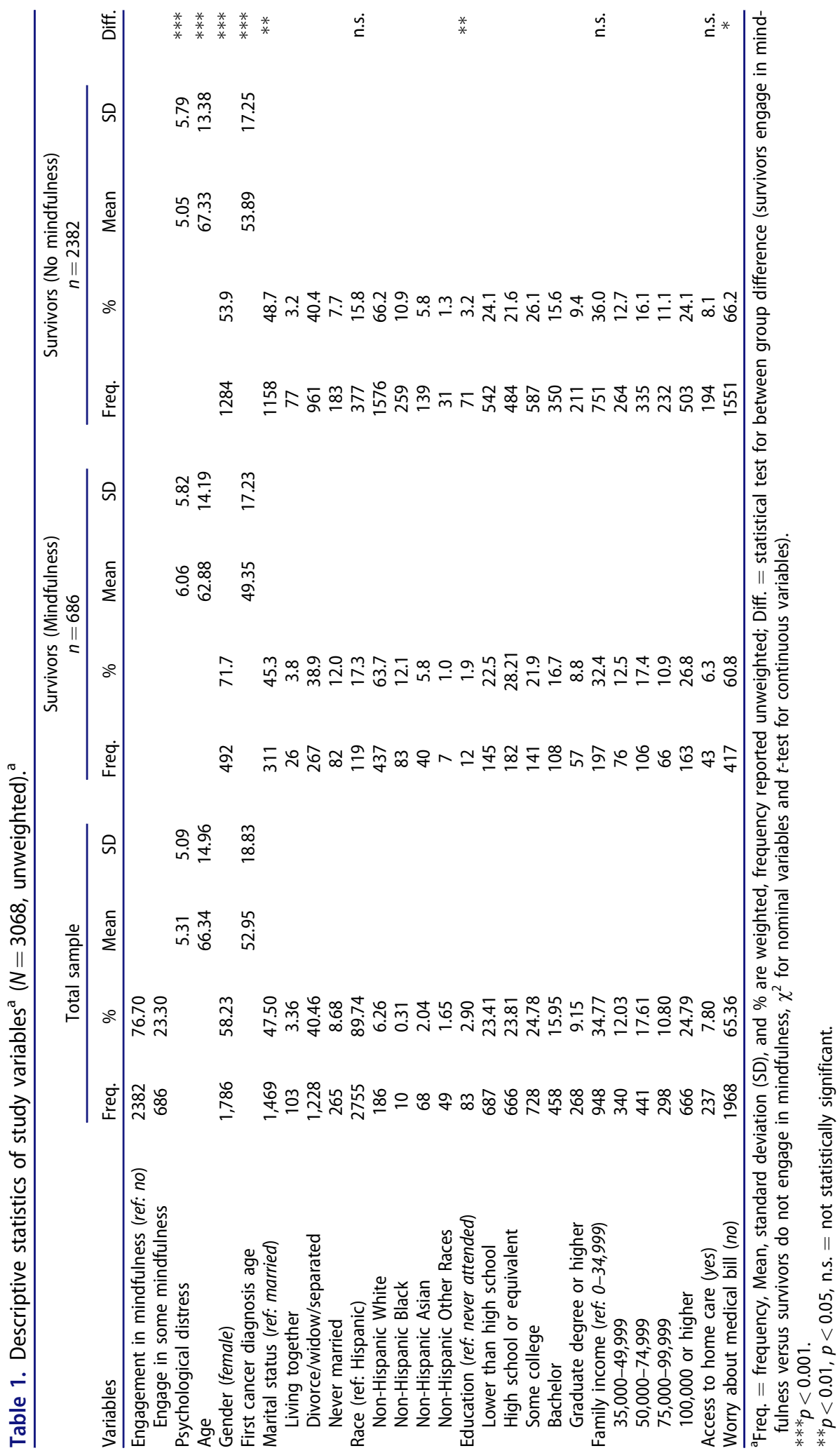




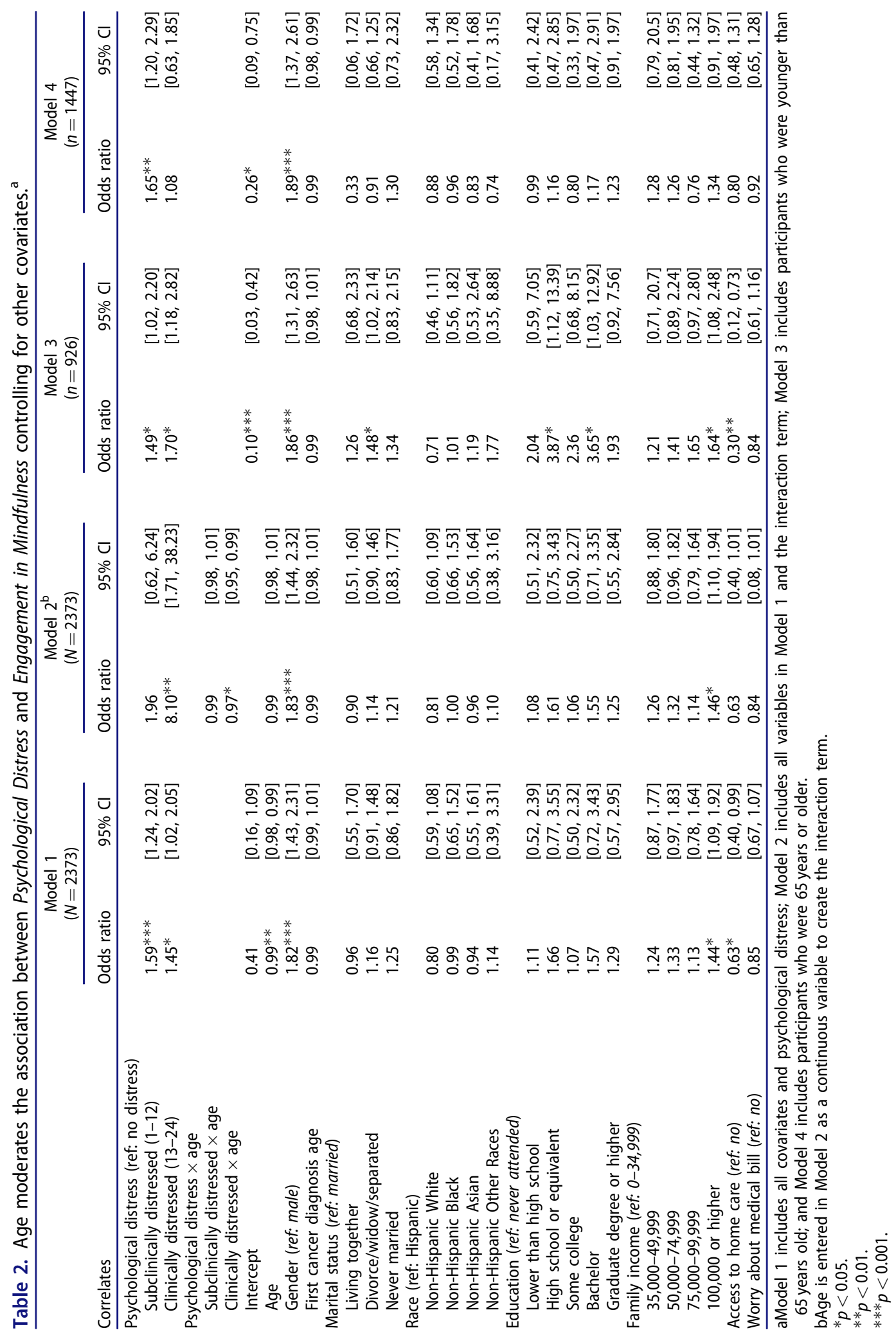


whereas those who were clinical distressed did not differ significantly from their non-distressed counterparts in mindfulness engagement, $O R=1.08, p>0.05$.

Further analysis was conducted to evaluate the moderating effect of age by splitting the sample into half (younger than 65 versus 65 years or older) and computing the weighted Pearson bivariate correlation between engagement in mindfulness and psychological distress (Table 3). Fisher's $r$-to- $z$ transformation indicated that the correlation coefficient between engagement in mindfulness and psychological distress was significantly greater among younger cancer survivors ( $<65$ years old), $r=0.137, p<0.001$ than their older counterparts (65 years or older), $r=0.054, p<0.05$. The difference between the coefficients was statistically significant, $z=2.24, p<0.05$.

Consistent across all four models, gender was a significant demographic variable associated with increased engagement in mindfulness. In comparison to a male cancer survivor, a female cancer survivor is 1.82 times more likely to engage in mindfulness, $b=1.82, p<0.001$. Notably, such gender difference was consistent for both younger and older cancer survivors, $b=1.86, p<0.001$, and $b=1.89, p<0.001$, respectively.

\section{Discussion}

Building on numerous clinical trials examining the relationship between mindfulness-based interventions and cancer survivors' psychological distress, to our knowledge, this study was the first to investigate the association between psychological distress and engagement in mindfulness using a national representative sample of cancer survivors. Both study hypotheses are supported by our analyses: (1) cancer survivors with greater psychological distress reported greater likelihood of engagement in mindfulness based practices, and (2) this association was much stronger among younger cancer survivors than their older counterparts.

Overall, we found a significant association between greater psychological distress and an increased likelihood of engaging in mindfulness-based practices. This finding suggests that, at the national level, there is increased awareness of using mindfulness-based practices to cope with psychological distress among cancer survivors. However, given the cross-sectional nature of the data, the relationship between psychological distress and engagement in mindfulness-based practices is associational rather than causal. Therefore, the timeframes for these two items may be more complex than we hypothesized here. For example, a cancer survivor may use mindfulness-based practices to cope with relatively low level of psychological distress. Yet, if the practice does not reduce distress effectively, a survivor may increase the frequency of practice. If it still does not work, it may incite 
Table 3. Weighted correlation coefficient $(r)$ between psychological distress and engagement in mindfulness.

\begin{tabular}{lllc}
\hline & & $r$ & $z$ \\
\hline Age & $<65$ years old & & \\
$(n=1148)$ & $0.137^{* * *}$ & $2.24^{*}$ \\
& 65 years old \\
$(n=1793)$ & $0.054^{*}$ & \\
\hline
\end{tabular}

$* * * p<0.001$.

${ }^{*} p<0.05$.

greater level of distress. In such scenario, it is actually the increased engagement in mindfulness-based practice that is "causing" a survivor's higher level of psychological distress.

In addition to the significant association between cancer survivors' psychological distress and engagement in mindfulness-based practices, we also revealed age as a significant moderator to this association. Consistent with the Motivational Theory of Life Span Development (Motivational Theory), ${ }^{11}$ we expect older cancer survivors in general to have developed better secondary control strategies than their younger counterparts. Therefore, older cancer survivors may pursue alternative secondary strategies (rather than mindfulness) to cope with their psychological distress. This idea was supported by our finding that association between psychological distress was significantly weaker among geriatric cancer survivors when compared with their younger counterparts. Such finding provides significant practice and research implications for psychosocial oncology practices. Knowing geriatric cancer survivors have become the largest population of US cancer survivors, their unique psychosocial challenges should be addressed in ways that are appropriate to their developmental characteristics. While mindfulness has been recognized as a powerful tool for distress reduction among cancer survivors, results of this study suggested that the likelihood of using mindfulness-based practices differs significantly by age among individuals with the same level of psychological distress. Therefore, psychosocial oncology providers should be aware of how different age groups of cancer survivors can respond differently to mindfulness practices.

A systematic review of 22 clinical trials on mindfulness-based therapy for cancer survivors reported cancer survivors' age averaged at 55 years old and ranged from 48 to 67 years old, ${ }^{3}$ indicating that research evidence regarding mindfulness for adult cancer survivors has only been supported among those who were younger than 65 years old but not for the geriatric cancer population. Results of this current study may provide one possible explanation to this age discrepancy that is older cancer survivors are less likely to engage in mindfulness practices and to be recruited in clinical trials. 
Alternative secondary control strategies that could further explain the positive relationship between mindfulness utilization and psychological distress among younger cancer survivors ( $<65$ years old) versus their older counterparts $(\geq 65)$, such as goal adjustment strategies. Theoretical models have proposed that people cope with goal disturbance or disruption via goal adjustment, and that employing goal adjustment strategies is associated with aging. ${ }^{11,30}$ Among newly diagnosed colorectal survivors, goal adjustment was found to be a common response to a cancer diagnosis, with the type of strategy used, transitioning from short-term to long-term over time. ${ }^{31}$ In this study neither age nor cancer stage was correlated with use of goal adjustment strategies, indicating that goal adjustment as a coping mechanism was consistent across the sample. Opposed to an alternative secondary control strategy, mindfulness could be utilized to support goal adjustment strategies among geriatric survivors.

In addition to the age disparity of utilizing mindfulness among distressed cancer survivors, this study also identified gender as a significant demographic variable with female survivors reporting greater likelihood of engaging in mindfulness. This finding aligns with the gender difference in relation to mindfulness practices among the general US population, ${ }^{32}$ and further highlights the importance of accounting for cancer survivors' demographic background when delivering mindfulness-based practices. It is critical for future clinical oncology research to further evaluate: (1) specific reasons why older cancer survivors were less likely to engage in mindfulness practices, (2) specific reasons why female cancer survivors were more likely to engage in mindfulness practices, (3) efficacy/effectiveness of mindfulness based practices for psychological distress among geriatric cancer survivors, and (4) the association between the efficacy/effectiveness and willingness to engage in mindfulness-based practices among geriatric cancer survivors.

\section{Strengths and limitations}

Several limitations of this study should be noted. First, this study is crosssectional in nature, thus limiting the causal relationship of the results. Second, while we controlled for participants' initial age of cancer diagnosis, we did not control for participants' specific cancer diagnosis because that variable is not available. Thirdly, the majority of our analytical sample is Hispanic. This is likely an artifact of the dataset's oversampling of Hispanic participants at the individual level as well as implementing heavier weights for Hispanic participants to create a nationally representative sample. Fourth, given the nature of the dataset, older adults who are institutionalized, e.g., in long-term care facilities, were not included in this study. This 
exclusion may potentially impact our study findings and deserve further investigation through connecting multiple national representative datasets that include institutionalized and non-institutionalized cancer patients. Finally, we used a subsample of a national representative sample rather than a cancer-specific dataset. While such decision strengthened the generalizability of our findings, future efforts should also investigate similar topics using a cancer-specific dataset to see if our findings persist.

Notwithstanding those above mentioned limitations, to our knowledge, this study is the first to evaluate the association between psychological distress and engagement in mindfulness among cancer survivors using a national representative sample. We also advanced literature by evaluating the moderating role of age to such relationship. Our findings emphasize the clinical importance of engaging in different service strategies when working with geriatric cancer in comparison to their younger counterparts. This is especially the case when utilizing mindfulness-related service which is a widely used and supported method.

\section{Acknowledgment}

This study has no funding to acknowledge.

\section{Disclosure statement}

No potential conflict of interest was reported by the authors.

\section{ORCID}

Anao Zhang (D) http://orcid.org/0000-0002-3199-1113

Kaipeng Wang (D) http://orcid.org/0000-0002-2206-4318

\section{Data availability statement}

This study used data from the 2017 National Health Interview Survey (NHIS): https://www. cdc.gov/nchs/nhis/index.htm

\section{References}

1. Noone AM, Howlader N, Krapcho M, et al. (eds). SEER Cancer Statistics Review. Bethesda, MD: National Cancer Institute; 2018.

2. Kwak M, Zebrack BJ, Meeske KA, et al. Trajectories of psychological distress in adolescent and young adult patients with cancer: a 1-year longitudinal study. JCO. 2013; 31(17):2160-2166. doi:10.1200/JCO.2012.45.9222

3. Piet J, Wurtzen H, Zachariae R. The effect of mindfulness-based therapy on symptoms of anxiety and depression in adult cancer patients and survivors: a systematic 
review and meta-analysis. J Consult Clin Psychol. 2012;80(6):1007-1020. doi:10.1037/ a0028329

4. Deimling GT, Bowman KF, Sterns S, et al. Cancer-related health worries and psychological distress among older adult, long-term cancer survivors. Psychooncology. 2006; 15(4):306-320. doi:10.1002/pon.955

5. Reed SC, Bell JF, Whitney R, et al. Psychosocial outcomes in active treatment through survivorship. Psycho-Oncol. 2018;27(1):279-285. doi:10.1002/pon.4444

6. Jacobsen PB. Clinical practice guidelines for the psychosocial care of cancer survivors current status and future prospects. Cancer. 2009;115(S18):4419-4429. doi:10.1002/ cncr.24589

7. Nishiura M, Tamura A, Nagai H, et al. Assessment of sleep disturbance in lung cancer patients: Relationship between sleep disturbance and pain, fatigue, quality of life, and psychological distress. Pall Supp Care. 2015;13(3):575-581. doi:10.1017/ S1478951513001119

8. Compen FR, Adang EMM, Bisseling EM, et al. Exploring associations between psychiatric disorder, psychological distress, and health care utilization in cancer patients (vol 27, pg 871, 2018). Psycho-Oncol. 2018;27(3):871-1671. doi:10.1002/pon.4591

9. Jones P, Blunda M, Biegel G, Carlson LE, Biel M, Wiener L. Can mindfulness-based interventions help adolescents with cancer? Psycho-Oncology. 2013;22(9):2148-2151. doi:10.1002/pon.3251

10. Gallegos AM, Hoerger M, Talbot NL, et al. Emotional benefits of mindfulness-based stress reduction in older adults: the moderating roles of age and depressive symptom severity. Aging Ment Health. 2013;17(7):823-829. doi:10.1080/13607863.2013.799118

11. Heckhausen J, Wrosch C, Schulz R. A motivational theory of life-span development. Psychol Rev. 2010;117(1):32-60. doi:10.1037/a0017668

12. Wrosch C, Schulz R, Miller GE, et al. Physical health problems, depressive mood, and cortisol secretion in old age: buffer effects of health engagement control strategies. Health Psychol. 2007;26(3):341-349. doi:10.1037/0278-6133.26.3.341

13. Boyle CC, Stanton AL, Ganz PA, et al. Improvements in emotion regulation following mindfulness meditation: effects on depressive symptoms and perceived stress in younger breast cancer survivors. J Consult Clin Psychol. 2017;85(4):397-402. doi:10. 1037/ccp0000186

14. Poulin PA, Romanow HC, Rahbari N, et al. The relationship between mindfulness, pain intensity, pain catastrophizing, depression, and quality of life among cancer survivors living with chronic neuropathic pain. Support Care Cancer. 2016;24(10): 4167-4175. doi:10.1007/s00520-016-3243-x

15. Lengacher CA, Johnson-Mallard V, Barta M, et al. Feasibility of a mindfulness-based stress reduction program for early-stage breast cancer survivors. J Holist Nurs. 2011; 29(2):107-117. doi:10.1177/0898010110385938

16. Branstrom R, Kvillemo P, Moskowitz JT. A randomized study of the effects of mindfulness training on psychological well-being and symptoms of stress in patients treated for cancer at 6-month follow-up. Intj Behav Med. 2012;19(4):535-542. doi:10. 1007/s12529-011-9192-3

17. Carlson LE, Beattie TL, Giese-Davis J, et al. Mindfulness-based cancer recovery and supportive-expressive therapy maintain telomere length relative to controls in distressed breast cancer survivors. Cancer. 2015;121(3):476-484. doi:10.1002/cncr.29063

18. Lengacher CA, Reich RR, Paterson CL, et al. Examination of broad symptom improvement resulting from mindfulness-based stress reduction in breast cancer 
survivors: a randomized controlled trial. JCO. 2016;34(24):2827-2834. doi:10.1200/ JCO.2015.65.7874

19. Mackenzie MJ, Carlson LE, Ekkekakis P, et al. Affect and mindfulness as predictors of change in mood disturbance, stress symptoms, and quality of life in a communitybased yoga program for cancer survivors. Evidence-Based Complement Alternat Med. 2013;2013:1-13. doi:10.1155/2013/419496

20. Huang HP, He M, Wang HY, et al. A meta-analysis of the benefits of mindfulnessbased stress reduction (MBSR) on psychological function among breast cancer (BC) survivors. Breast Cancer. 2016;23(4):568-576. doi:10.1007/s12282-015-0604-0

21. Zhang MF, Wen YS, Liu WY, et al. Effectiveness of mindfulness-based therapy for reducing anxiety and depression in patients with cancer a meta-analysis. Medicine. 2015;94(45):e0897. doi:10.1097/MD.0000000000000897

22. Lerner RM. Developmental regulation in adulthood: age-normative and sociostructural constraints as adaptive challenges. Contemp Psychol-Apa Rev Books. 1999;44(6): 453-455. doi:10.1037/002092

23. Wrosch C, Sabiston CM. Goal adjustment, physical and sedentary activity, and wellbeing and health among breast cancer survivors. Psycho-Oncol. 2013;22(3):581-589. doi:10.1002/pon.3037

24. Heckhausen J, Wrosch C. Challenges to developmental regulation across the life course: what are they and which individual differences matter?. Int J Behav Dev. 2016;40(2):145-150. doi:10.1177/0165025415588796

25. Statistics NCfH. National Health Interview Survey (NHIS) public use data release: Survey description. 2017.

26. Tomitaka S, Kawasaki Y, Ide K, et al. Characteristic distribution of the total and individual item scores on the Kessler Screening Scale for Psychological Distress (K6) in US adults. BMC Psychiatry. 2017;17(1):290. doi:10.1186/s12888-017-1449-1

27. Prochaska JJ, Sung HY, Max W, et al. Validity study of the K6 scale as a measure of moderate mental distress based on mental health treatment need and utilization. Int $J$ Methods Psychiatr Res. 2012;21(2):88-97. doi:10.1002/mpr.1349

28. Fisher RA. Statistical Methods for Research Workers. 14th ed. New York, NY: Hafner Publishing; 1973.

29. Moon M, Guo J, McSorley VE. Is 65 the best cutoff for defining "Older Americans". 2015.

30. Freund AM, Nikitin J, Ritter JO. Psychological consequences of longevity. Human Development. 2009;52(1):1-37. - doi:10.1159/000189213

31. Janse M, Fleer J, Smink A, et al. Which goal adjustment strategies do cancer patients use? A longitudinal study. Psycho-Oncology. 2016;25(3):332-338. doi:10.1002/pon. 3924

32. Olano HA, Kachan D, Tannenbaum SL, et al. Engagement in mindfulness practices by U.S. adults: sociodemographic barriers. J Altern Complement Med. 2015;21(2): 100-102. doi:10.1089/acm.2014.0269 